Tropical Journal of Pharmaceutical Research July 2019; 18 (7): 1419-1425

ISSN: 1596-5996 (print); 1596-9827 (electronic)

(1) Pharmacotherapy Group, Faculty of Pharmacy, University of Benin, Benin City, 300001 Nigeria.

\title{
Sulfanilamide benzotriazole tetrazole inhibits neuronal apoptosis in neonatal rats by targeting JNK and p38 MAPK pathways
}

\author{
Xiaolin Hu, Peng Xiong* \\ Department of Paediatrics, Tongji Hospital, Tongji Medical College, Huazhong University of Science and Technology, Wuhan, \\ Hubei 430030, China
}

*For correspondence: Email: patrils@yahoo.com; Tel/Fax: 0086-027-83662684

\begin{abstract}
Purpose: To investigate the neuroprotective effect of sulfanilamide benzotriazole tetrazole (SBT) in neonatal rats exposed to isoflurane, and also to elucidate the underlying mechanism.

Methods: Rat pups $(n=60)$ were randomly assigned to six groups of 10 pups each: normal control group, negative control group, $5 \mathrm{mg} / \mathrm{kg}$ SBT group, $10 \mathrm{mg} / \mathrm{kg}$ SBT group, $15 \mathrm{mg} / \mathrm{kg}$ SBT group, and 20 $\mathrm{mg} / \mathrm{kg}$ SBT group. With exception of normal control group, pups were exposed to isoflurane $(0.75 \%)$ for $6 \mathrm{~h}$ on postnatal day 7. The negative control group was not treated, while pups in the four treatment groups received 5, 10, 15 and $20 \mathrm{mg} / \mathrm{kg} \mathrm{SBT}$, respectively, $1 \mathrm{~h}$ after exposure to anaesthesia. TUNEL assay was used to determine the extent of apoptosis in cornu ammonis area-1 (CA-1), cornu ammonis area-3 (CA-3) and dentate gyrus of rat hippocampal tissues. Expressions of apoptotic and anti-apoptotic proteins were determined using Western blotting. Evaluation of learning and memorizing ability was done using Morris water maze test.

Results: Isoflurane significantly increased the extent of apoptosis in CA-1, CA-3 and dentate gyrus of rat hippocampal tissues $(p<0.05)$. However, treatment with SBT significantly and dose-dependently reduced neuronal apoptosis $(p<0.05)$. The expression of caspase 3 was significantly upregulated by isoflurane, but was significantly and dose-dependently down-regulated by SBT $(p<0.05)$. Isoflurane significantly increased Bax expression, and decreased the expression of bcl-2 $(p<0.05)$. The effects of isoflurane on the expression of these proteins were significantly and dose-dependently reversed by SBT $(p<0.05)$. The expression of $b c l x L$ in rat hippocampal tissues was significantly down-regulated by isoflurane, but was significantly and dose-dependently upregulated by SBT $(p<0.05)$. The escape latency of pups was significantly higher in negative control group than in normal control group, but SBT treatment significantly and dose-dependently reversed this trend $(p<0.05)$.

Conclusion: These results suggest that SBT prevents neuronal apoptosis, and improves the ability to learn and memorize in neonatal rats exposed to isoflurane via regulation of apoptotic, JNK and p38 MAPK protein expressions.
\end{abstract}

Keywords: Sulfanilamide benzotriazole tetrazole, Isoflurane, Neuronal apoptosis, Neuroprotection, Expression

\footnotetext{
This is an Open Access article that uses a fund-ing model which does not charge readers or their institutions for access and distributed under the terms of the Creative Commons Attribution License (http://creativecommons.org/licenses/by/4.0) and the Budapest Open Access Initiative (http://www.budapestopenaccessinitiative.org/read), which permit unrestricted use, distribution, and reproduction in any medium, provided the original work is properly credited.

Tropical Journal of Pharmaceutical Research is indexed by Science Citation Index (SciSearch), Scopus, International Pharmaceutical Abstract, Chemical Abstracts, Embase, Index Copernicus, EBSCO, African Index Medicus, JournalSeek, Journal Citation Reports/Science Edition, Directory of Open Access Journals (DOAJ), African Journal Online, Bioline International, Open-J-Gate and Pharmacy Abstracts
} 


\section{INTRODUCTION}

Tetrazoles are a class of synthetic organic heterocyclic compounds consisting of a 5membered ring containing four nitrogen atoms and a carbon atom. The SBT ring is highly preferred for designing drugs because it enhances their bioactivity in living systems [1]. Compounds bearing SBT ring have been reported to act as anti-convulsant, anti-inflammatory and antinociceptive agents [1]. The SBT scaffold attached to sulfanilamide benzotriazole exhibited better anti-inflammatory effects than that of the standard therapeutic molecule, paracetamol [2]. Moreover, the combination of tetrazole with sulfanilamide benzotriazole produced antinociceptive activity comparable with that of pentazocine [2].

Developing brains are very sensitive to anaesthesia, the chronic exposure of which leads to neuronal degeneration [3-5]. Loss of memory and cognition has been reported in animals exposed to chronic doses of anaesthetics [3-5]. Studies involving isoflurane anaesthesia have shown that long-term exposure causes neuronal apoptosis in the central nervous system (CNS) via activation of mitochondrial pathway pathway of apoptosis [6,7]. Isoflurane activates members of mitogen activated protein kinases (MAPKs) family such as JNK, p38 MAPK and ERKs [5]. Neuronal apoptosis stimulated by the JNK pathway is associated with suppression of bcl-2 expression and phosphorylation of c-Jun [8]. It has been shown that MAPK and JNK pathways are associated with neuronal degeneration in the hippocampal region of rats [5].

Cognitive impairment in postoperative patients below 4 years of age has been reported $[9,10]$. Reports on the harmful effect of anaesthesia and its ability to cause neuronal degeneration during developmental stage abound. Therefore, it has become necessary to develop new treatment strategies that can effectively protect patients against anaesthesia-induced damage to the nervous system.

The present study investigated the neuroprotective effect of SBT in neonatal rats exposed to isoflurane, and the underlying mechanism.

\section{EXPERIMENTAL}

\section{Materials}

Fluorometric dead end TUNEL kit was purchased from Promega Corporation (USA); protein extraction kit was obtained from Bio-Rad Biotechnology; and bicinchoninic (BCA) protein assay kits were product of Beijing Solarbio Science \& Technology Co. Ltd. (China). Enhanced chemiluminescence (ECL) system was obtained from Santa Cruz Biotechnology, Inc. (USA), while PVDF membranes were purchased from Roche Diagnostics. National Institute of Health image 1.41 software was a product of Bethesda (USA). Rabbit polyclonal anticaspase-3, bcl-xL, bad, p-bad, bax, bcl-2, ERK1/2, p-ERK1/2, JNK, p-JNK, p-c-Jun, p38, $p$-p38, and $\beta$-actin were products of Abcam (USA), while ANY-maze was obtained from Stoelting Co., Ltd. (USA).

\section{Rats}

Pregnant Sprague Dawley rats $(\mathrm{n}=12)$ were obtained from the Animal Centre of Shandong University, Jinan, China. The rats were housed in metal cages under controlled conditions of $12 \mathrm{~h}$ light/12 h dark cycle at $24{ }^{\circ} \mathrm{C}$ and humidity of 55 $60 \%$. They were allowed free access to standard rat feed and clean water. The rats littered sixty pups which were housed separately in plastic cages under standard conditions. Approval for this study was obtained from the Ethics Committee for the Use and Care of Animals of Huazhong University of Agriculture (No. HAU/16/2067). The experimental procedures were based on the guidelines for Laboratory Animal Use, National Institutes of Health [11].

\section{Treatment regimen}

The rat pups were randomly assigned to six groups of 10 pups each: normal control group, negative control group, $5 \mathrm{mg} / \mathrm{kg}$ bwt SBT group, $10 \mathrm{mg} / \mathrm{kg}$ bwt SBT group, $15 \mathrm{mg} / \mathrm{kg}$ bwt SBT group and $20 \mathrm{mg} / \mathrm{kg}$ bwt SBT group. With exception of normal control group, the pups were exposed to isoflurane $(0.75 \%)$ for $6 \mathrm{~h}$ on postnatal day 7 . The negative control group was not treated, while pups in the 4 treatment groups received $5,10,15$ and $20 \mathrm{mg} / \mathrm{kg}$ bwt SBT, respectively, $1 \mathrm{~h}$ after exposure to anaesthesia.

\section{Sample collection}

The pups were sacrificed on postnatal day 15 and their brains were excised using transcardial perfusion with ice-cold normal saline. The tissues were fixed in phosphate-buffered paraformaldehyde (4\%). Neuronal apoptosis and protein expression in pups hippocampal tissues were then determined. The pups were also subjected to analysis of memorising and learning ability on postnatal day 31 . 


\section{Neuronal apoptosis assay}

Terminal deoxynucleotidyl transferase dUTP nick end labeling (TUNEL) assay was used for the determination of apoptosis in pups hippocampal tissues. The excised brain tissues were fixed in formaldehyde at $4^{\circ} \mathrm{C}$ for $5 \mathrm{~h}$ and subsequently embedded in paraffin. The embedded tissues were then sliced into thin $2 \mu \mathrm{m}$ sections using refrigerated microtome. Fluorimetric dead end TUNEL kit was used for the determination of TUNEL-positive cell population, while inverted Eclipse Ti2 microscope was used for analysis of TUNEL- positive cells in CA-1, CA-3 and dentate gyrus.

\section{Western blotting}

The pups were euthanized and their hippocampal tissues were isolated. Cell suspension resulting from trypsinization of the tissues was washed twice with phosphate-buffered saline (PBS) and lysed with ice-cold radio-immunoprecipitation assay buffer (RIPA) containing protease inhibitor. The resultant lysate was centrifuged at 15, 000 rpm for $15 \mathrm{~min}$ at $4{ }^{\circ} \mathrm{C}$, and the protein concentration of the supernatant was determined using bicinchoninic (BCA) assay kit. A portion of total cell protein $(30 \mu \mathrm{g})$ from each sample was separated on a $12 \%$ sodium dodecyl sulphate (SDS)-polyacrylamide gel electrophoresis and transferred to a fixed polyvinylidene fluoride membrane at $110 \mathrm{~V}$ and $90{ }^{\circ} \mathrm{C}$ for $120 \mathrm{~min}$. Subsequently, non-fat milk powder $(5 \%)$ in Trisbuffered saline containing $0.2 \%$ Tween-20 (TBST) was added with gentle shaking at $37{ }^{\circ} \mathrm{C}$ and incubated to block non-specific binding of the blot.

The blots were incubated overnight at $4{ }^{\circ} \mathrm{C}$ with primary antibodies of rabbit polyclonal anticaspase-3, bcl-xL, bad, p-bad, bax, bcl-2, ERK1/2, p-ERK1/2, JNK, p-JNK, p-c-Jun, p38, $\mathrm{p}$-p38, and $\beta$-actin at a dilution of 1 to 500 . Then, the membrane was washed thrice with TBS-T and further incubated with horseradish peroxidaseconjugated goat anti-rabbit IgG secondary antibody for $2.5 \mathrm{~h}$ at room temperature. The blot was developed using an X-ray film. Grayscale analysis of the bands was performed using ECL. The various protein expression levels were normalized to that of $\beta$-actin which was used as a standard.

\section{Morris water maze test}

The escape latency of pups was determined using Morris water maze test. Pups were given training from postnatal day 25 to day 28 using the Morris water maze. A platform of $10 \mathrm{~cm}$ diameter was placed in a circular water pool of diameter $210 \mathrm{~cm}$ and depth $64 \mathrm{~cm}$. The temperature of the water in the pool was maintained at $24 \pm 2{ }^{\circ} \mathrm{C}$. The pups were put into the pool twice daily and allowed to locate the submerged platform within $60 \mathrm{sec}$. The pups that could not locate the platform in $60 \mathrm{sec}$ were guided towards it.

The time taken (latency time) by the pups to locate the platform was calculated using video tracking system (ANY-maze). After the completion of trial, the pups were warmed using infrared lamp in holding cages before being transferred to their cages.

\section{Analysis of cognitive impairment}

Impairment of cognitive behaviour was determined in the pups on postnatal day 30 using cued recall test. The pups were put into a particular portion of the swimming pool close to the end. A white cloth was used to cover the pool completely to prevent any visual cue. A rod placed around $20 \mathrm{~cm}$ above the water in any one of the 40 quadrants randomly served as a cue to enable the pups locate the platform. Pups were given four chances $60 \mathrm{sec}$ each to find platform and then rest on it for another $30 \mathrm{sec}$. Pups that could not find the platform were guided towards it and allowed to take $30 \mathrm{sec}$ rest. After 4-trials, the pups were removed.

\section{Place trials}

The pools were uncovered after completion of cued recall test and the pups were again put in any one of the quadrants. The time taken by a pup to reach the platform in the absence of the cue rod and in the presence of the rod was recorded. Pups were placed in the same position throughout the trials to determine the time taken to reach the platform.

\section{Probe trials}

After $24 \mathrm{~h}$ of completion of place trials, memorising ability of the pups was determined using probe trials. The pups were put in opposite quadrant and the platform in the pool was removed. The rats were given $60 \mathrm{sec}$ to make attempts to locate the position of the submerged platform.

\section{Statistical analysis}

Data are expressed as mean $\pm S D$, and the statistical analysis was performed using SPSS (17). Groups were compared using Duncan's multiple range test. Values of $p<0.05$ were considered statistically significant. 


\section{RESULTS}

\section{Inhibition of neuronal apoptosis by SBT}

Isoflurane significantly increased the extent of apoptosis in the CA-1, CA-3 and dentate gyrus of rat hippocampal tissues $(p<0.05)$. TUNEL-positive cell population was significantly higher in CA-1, CA-3 and dentate gyrus of rats exposed to isoflurane than in normal control group $(p<0.05)$. However, treatment with SBT significantly and dose-dependently reduced neuronal apoptosis $(p<0.05$; Figure 1$)$.

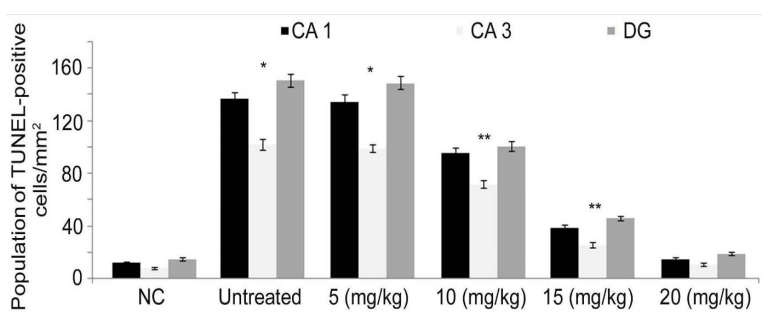

Figure 1: Inhibition of neuronal apoptosis by SBT as revealed using TUNEL-assa; $p<0.05,{ }^{*} p<0.01$, when compared with negative control group

\section{Degree of expressions of apoptotic and anti- apoptotic proteins in rat hippocampal tissues}

The expression of caspase-3 was significantly upregulated by isoflurane, but was significantly and dose-dependently down-regulated by SBT ( $p$ $<0.05$ ). Isoflurane also significantly increased the expression of bax and decreased the expression of bcl-2 $(p<0.05)$. The effects of isoflurane on the expression of these proteins were significantly and dose-dependently reversed by SBT $(p<$ $0.05)$. The expression of bcl-xL in rat hippocampal tissues was significantly down-regulated by isoflurane, but was significantly and dosedependently upregulated by SBT $(p<0.05)$. Isoflurane significantly increased the expression of bad and significantly decreased the expression of $p$-bad $(p<0.05)$. However, treatment with SBT significantly reversed the effects of isoflurane on the expressions of these proteins $(p<0.05)$. These results are shown in Figures $2 \mathrm{~A}$ and 2B.
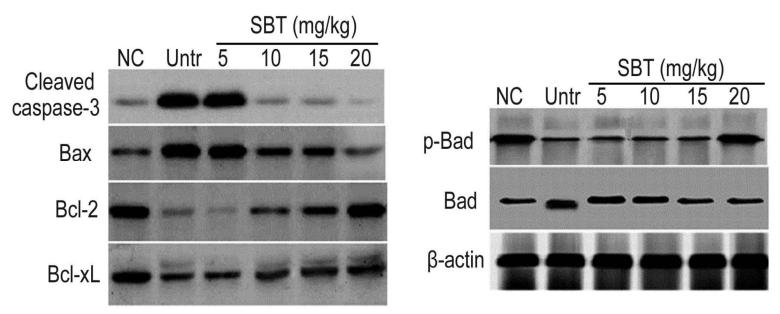

Figure 2: Expressions of apoptotic and anti-apoptotic proteins as measured using Western blotting. ${ }^{*} P<0.05$, when compared with negative control group
Expression of JNK, c-Jun, ERK and p38 in neonatal rats

Isoflurane upregulated the expression of JNK, $c$-Jun, ERK and p38, and their phosphorylated forms in hippocampal tissues of neonatal rats $(p<$ 0.05). However, treatment with SBT significantly and dose-dependently down-regulated the expression of these proteins and their phosphorylated forms $(p<0.05)$. Treatment with SBT also significantly inhibited isoflurane-induced phosphorylation of ERK1/2 $(p<0.05$; Figure 3$)$.

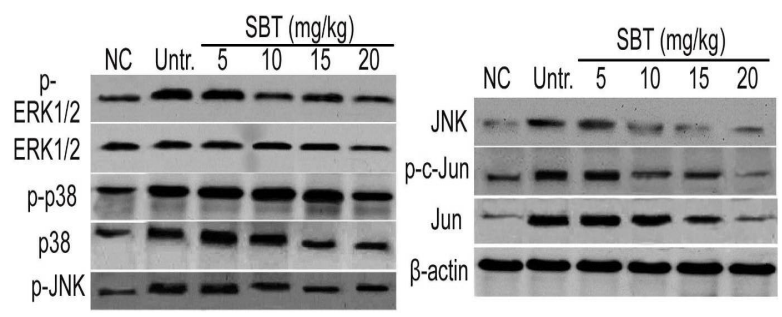

Figure 3: Expression of MAPK pathway proteins. ${ }^{*} P<$ 0.05 , when compared with negative control group

\section{Effect of SBT on isoflurane-induced changes in memory and behaviour of neonatal rats}

The escape latency of pups in negative control group was significantly higher than that of pups in normal control group $(p<0.05)$. However, treatment with SBT significantly and dosedependently reduced the escape latency of the pups $(p<0.05$; Figure 4$)$.

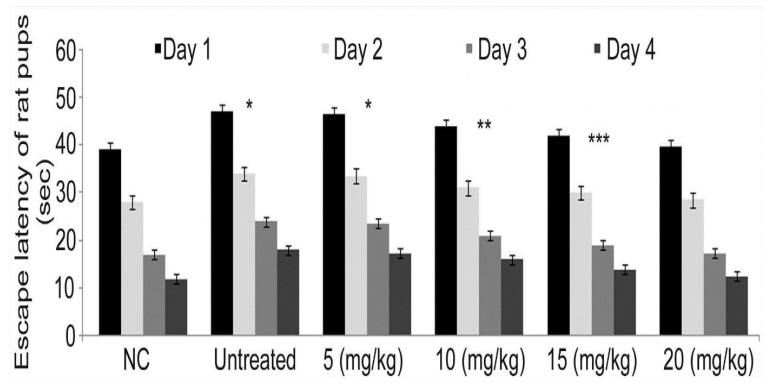

Figure 4: Measurement of escape latency in rats. ${ }^{\prime} P<$ $0.05,{ }^{* *} p<0.01$ and ${ }^{* * *} p<0.001$, when compared with negative control group

\section{Effect of SBT on the ability of rat pups to learn and memorize}

Exposure of neonatal rats to isoflurane significantly increased the time taken by them to reach the submerged platform, when compared with normal control group $(p<0.05)$. However, treatment with SBT significantly and dosedependently decreased the time taken to reach the submerged platform $(p<0.05)$. The results from place and probe tests showed that neonatal rats exposed to isoflurane took significantly longer 
time to reach the new platform than those of normal control group $(p<0.05)$. Pups treated with SBT reached the new platform faster than those in the negative control group $(p<0.05)$.

The time spent in target quadrant searching for platform was significantly less in negative control group than in the normal control group $(p<0.05)$. Treatment with SBT significantly and dosedependently increased the time spent in target quadrant searching for platform $(p<0.05)$. The rats treated with $20 \mathrm{mg} / \mathrm{kg}$ bwt SBT spent almost equal time in target quadrant as those of normal control group. These results are shown in Figure 5 .

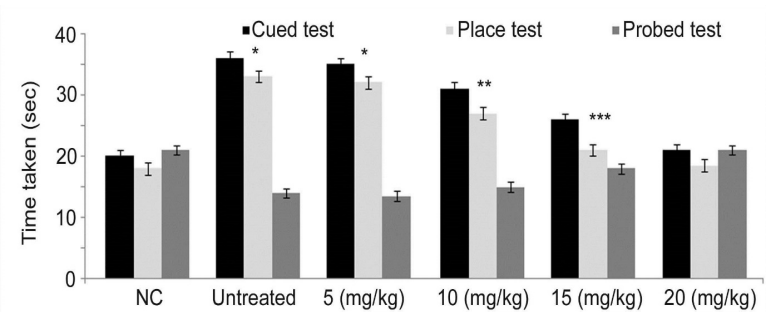

Figure 5: Evaluation of the ability of neonatal rats to learn and memorise. ${ }^{*} P<0.05, \quad p<0.01$ and ${ }^{* \star *} p<$ 0.001 , when compared with negative control group

\section{DISCUSSION}

The present study investigated the neuroprotective effect of SBT in neonatal rats exposed to isoflurane, and the underlying mechanism. The results showed that SBT prevented apoptosis of neurons, and improved the ability of neonatal rats to learn and memorise even when exposed to isoflurane. Studies have shown that neonatal rats experience neuronal damage which leads to memory loss and cognitive impairment on exposure to an anesthetic compound such as isoflurane [12].

Brain development and maturation is associated with the elimination of approximately $60 \%$ progenitor and neuronal cells through apoptosis [13]. It has been reported that anesthesia and pathological factors enhance neuronal apoptosis beyond that normally required for development [14]. In the developing brain of neonates, neuronal degeneration is usually induced via exposure to isoflurane $[3,5]$. In this study, neuronal apoptosis in CA-1, CA-3 and dentate gyrus was significantly increased on exposure to isoflurane. Treatment of isoflurane exposed pups with SBT significantly reduced neuronal apoptosis.

Caspase-3 plays an important role in the induction of apoptosis [15]. The expression of cleaved caspase- 3 is considered as a marker of cell death via the apoptotic pathway $[3,5,13]$. In this study, the expression of caspase-3 was significantly upregulated by isoflurane, but was significantly and dose-dependently down-regulated by SBT. The expression of bcl-xL in tissues of the nervous system is generally high, since it maintains survival of the neurons by regulating integrity of the mitochondrial membrane, thereby preventing cytochrome $c$ release [16]. In this study, exposure of neonatal rats to isoflurane significantly downregulated the expression of bcl-xL in hippocampal tissues, when compared with normal control group. However, treatment with SBT significantly reversed the effect of isoflurane.

Members of bcl-2 gene family regulate the expressions of pro-apoptotic and anti-apoptotic proteins [17]. A slight imbalance in the expressions of bcl-2 proteins causes cytochrome $c$ release and activation of caspase-3, thereby inducing apoptosis [17]. The results of this study showed that isoflurane significantly increased the expression of bax and decreased the expression of bcl-2. The effects of isoflurane on the expressions of these proteins were significantly and dose-dependently reversed by SBT. The expression of bcl-xL in rat hippocampal tissues was significantly down-regulated by isoflurane, but was significantly and dose-dependently upregulated by SBT.

Isoflurane significantly increased the expression of bad and significantly decreased the expression of p-bad. However, treatment with SBT significantly reversed the effects of isoflurane on the expression of these proteins. These results suggest that treatment with tetrazole may effectively regulate the expression of bcl-2 protein in the hippocampal tissues of neonatal rats. Studies have shown that neuronal degeneration due to anesthesia exposure is associated with the activation of MAPK and JNK pathways [18]. The activation of JNK leads to phosphorylation of c-Jun which in turn causes transcription of apoptosis-related genes [19].

In this study, isoflurane upregulated the expressions of JNK, c-Jun, ERK and p38, and their phosphorylated forms in hippocampal tissues of neonatal rats. However, treatment with SBT significantly and dose-dependently downregulated the expression of these proteins and their phosphorylated forms. Treatment with SBT significantly inhibited isoflurane-induced phosphorylation of ERK $1 / 2$. These results suggest that SBT may prevent neuronal apoptosis induced by isoflurane in neonatal rats via down-regulation of p38 MAPK. It has been reported that exposure of rats to isoflurane causes activation of p38 MAPK in the nervous system [20]. It is likely that SBT inhibits the expressions of anti-apoptotic 
proteins, and promotes apoptotic protein expressions in neonatal rats.

Exposure of rodents to anaesthesia decreases learning and memorising ability $[3,4]$. Learning disabilities are associated with damage to hippocampal tissues [21]. In this study, escape latency of pups in the negative control group was significantly higher than that of pups in the normal control group. However, treatment with SBT significantly and dose-dependently reduced the escape latency of the pups, an indication that SBT may inhibit hippocampal tissue damage and ultimately prevent memory loss.

\section{CONCLUSION}

The results obtained in this study suggest that SBT prevents neuronal apoptosis, and improves the learning and memory of neonatal rats exposed to isoflurane via regulation of expressions of apoptotic, JNK and p38 MAPK proteins.

\section{DECLARATIONS}

\section{Contribution of authors}

We declare that this work was done by the authors named in this article and all liabilities pertaining to claims relating to the content of this article will be borne by the authors. Xiaolin Hu performed the experimental work, carried out the literature survey and analysed the data. Peng Xiong compiled the data, designed the study and wrote the paper. Both the authors read the paper thoroughly and approved it for publication.

\section{Open Access}

This is an Open Access article that uses a funding model which does not charge readers or their institutions for access and distributed under the terms of the Creative Commons Attribution License (http://creativecommons.org/licenses/by/ 4.0) and the Budapest Open Access Initiative (http://www.budapestopenaccessinitiative.org/rea d), which permit unrestricted use, distribution, and reproduction in any medium, provided the original work is properly credited.

\section{REFERENCES}

1. Dai LL, Cui SF, Damu GLV, Zhou CH. Synthesis and advance in research of SBTs. Chin J Org Chem 2013; 33: 224-244.
2. Rajasekaran A, Rajagopal KA. Synthesis of some novel triazole derivatives as anti-nociceptive and antiinflammatory agents. Acta Pharm 2009; 59: 355-364.

3. Jevtovic Todorovic $V$, Hartman RE, Izumi $Y$, Benshoff ND, Dikranian K, Zorumski CF, Olney JW, Wozniak DF. Early exposure to common anesthetic agents causes widespread neurodegeneration in the developing rat brain and persistent learning deficits. $J$ Neurosci 2003; 23: 876882.

4. Satomoto M, Satoh $Y$, Terui K, Miyao H, Takishima K, Ito $M$, Imaki J. Neonatal exposure to sevoflurane induces abnormal social behaviors and deficits in fear conditioning in mice. Anesthesiology 2009; 110: 628 637.

5. Brambrink AM, Evers AS, Avidan MS, Farber NB, Smith $D J$, Zhang $X$, Dissen GA, Creeley CE, Olney JW. Isoflurane induced neuroapoptosis in the neonatal rhesus macaque brain. Anesthesiology 2010; 112: 834 841.

6. Wei $H$, Kang B, Wei W, Liang G, Meng QC, Li Y, Eckenhoff RG. Isoflurane and sevoflurane affect cell survival and BCL 2/BAX ratio differently. Brain Res 2005; 1037: 139147.

7. Yon JH, Daniel Johnson J, Carter LB, Jevtovic Todorovic $V$. Anesthesia induces neuronal cell death in the developing rat brain via the intrinsic and extrinsic apoptotic pathways. Neuroscience 2005; 135: 815827.

8. Behrens A, Sibilia M, Wagner EF. Amino terminal phosphorylation of $C$ Jun regulates stress induced apoptosis and cellular proliferation. Nat Genet 1999; 21 : 326329.

9. DiMaggio C, Sun LS, Li G. Early childhood exposure to anesthesia and risk of developmental and behavioral disorders in a sibling birth cohort. Anesth Analg 2011; 113: 11431151.

10. Ing C, DiMaggio C, Whitehouse A, Hegarty MK, Brady J, von Ungern Sternberg BS, Davidson A, Wood AJ, Li G, Sun LS. Long term differences in language and cognitive function after childhood exposure to anesthesia. Pediatrics 2012; 130: e476 e485.

11. Garber JC. Committee for the Update of the Guide for the Care and Use of Laboratory Animals. Guide for the Care and Use of Laboratory Animals, 8th ed. National Academy of Sciences, 2011.

12. Liang G, Ward C, Peng J, Zhao $Y$, Huang B, Wei $H$. Isoflurane causes greater neurodegeneration than an equivalent exposure of sevoflurane in the developing brain of neonatal mice. Anesthesiology 2010; 112: 1325 1334.

13. Oppenheim RW. Cell death during development of the nervous system. Annu Rev Neurosci 1991; 14: 453501.

14. Blomgren K, Leist M, Groc L. Pathological apoptosis in the developing brain. Apoptosis 2007; 12: 9931010.

15. Gown AM, Willingham MC. Improved detection of apoptotic cells in archival paraffin sections: Immunohistochemistry using antibodies to cleaved caspase 3. J Histochem Cytochem 2002; 50: 449454. 
16. Zhao $H$, Yenari MA, Cheng D, Sapolsky RM, Steinberg $G K . B c l 2$ overexpression protects against neuron loss within the ischemic margin following experimental stroke and inhibits cytochrome $c$ translocation and caspase 3 activity. J Neurochem 2003; 85: 10261036.

17. Chu R, Upreti $M$, Ding WX, Yin XM, Chambers TC. Regulation of Bax by $\mathrm{c}$ Jun $\mathrm{NH} 2$ terminal kinase and $\mathrm{BCl}$ $x L$ in vinblastine induced apoptosis. Biochem Pharmacol 2009; 78: 241248.

18. Wang WY, Yang R, Hu SF, Wang H, Ma ZW, Lu Y. N stearoyl I tyrosine ameliorates sevoflurane induced neuroapoptosis via MEK/ERK1/2MAPK signaling pathway in the developing brain. Neurosci Lett 2013; 541: 167172
19. Guan $Q H$, Pei DS, Zhang QG, Hao ZB, Xu TL, Zhang $G Y$. The neuroprotective action of SP600125, a new inhibitor of JNK, on transient brain ischemia/reperfusion induced neuronal death in rat hippocampal CA1 via nuclear and non-nuclear pathways. Brain Res 2005; 1035: 5159.

20. Zheng S, Zuo Z. Isoflurane preconditioning induces neuroprotection against ischemia via activation of $P 38$ mitogen activated protein kinases. Mol Pharmacol 2004; 65: 11721180.

21. Morris RG, Garrud P, Rawlins JN, O'Keefe J. Place navigation impaired in rats with hippocampal lesions. Nature 1982; 297: 681683. 